\title{
Investigation of pharmacological responses to anti-diabetic drugs in female Spontaneously Diabetic Torii (SDT) fatty rats, a new nonalcoholic steatohepatitis (NASH) model
}

\author{
Yasufumi TORINIWA ${ }^{1)}$, Tomoyuki SAITO ${ }^{1)}$, Katsuhiro MIYAJIMA ${ }^{1,2)}$, \\ Yukihito ISHII'), Kinuko UNO ${ }^{2)}$, Tatsuya MAEKAWA ${ }^{1)}$, Tohru MATSUI'), \\ Shinichi KUME ${ }^{3)}$, Takahisa YAMADA ${ }^{4)}$ and Takeshi OHTA ${ }^{1) *}$ \\ 1)Central Pharmaceutical Research Institute, Japan Tobacco Inc., 1-1 Murasaki-cho, Takatsuki, Osaka 569-1125, \\ Japan \\ ${ }^{2)}$ Department of Nutritional Science and Food Safety, Faculty of Applied Biosciences, Tokyo University of \\ Agriculture, 1-1-1 Sakuragaoka, Setagaya-ku, Tokyo 156-8502, Japan \\ 3)Kyoto University, Graduate School of Agriculture, Kitashirakawa, Sakyo-ku, Kyoto 606-8502, Japan \\ 4) Laboratory of Animal Genetics, Graduate School of Science and Technology, Niigata University, \\ 2-8050 Ikarashi, Nishi-ku, Niigata 950-2181, Japan
}

J. Vet. Med. Sci.

80(6): 878-885, 2018

doi: 10.1292/jvms.18-0119

Received: 6 March 2018

Accepted: 2 April 2018

Published online in J-STAGE:

10 April 2018
ABSTRACT. Nonalcoholic steatohepatitis (NASH) is a progressive liver disease, and some patients develop hepatic cirrhosis/carcinoma. Animal models play key roles in the development of new therapies for NASH. In this study, the pharmacological effects of metformin and pioglitazone were investigated in female Spontaneously Diabetic Torii (SDT) fatty rats to verify the utility of this model. The anti-diabetic drugs were administered to SDT fatty rats fed a cholesterol-enriched diet from 4 to 25 weeks, and changes in food intake, body weight, and blood chemistry parameters were evaluated every 4 weeks. The hepatic lipid content, mRNA expression in relation to lipid synthesis, inflammation, and fibrosis, and histopathological analyses were performed at 25 weeks. Pioglitazone improved hyperglycemia, hyperlipidemia, and abnormalities in hepatic parameters. The insulin levels were lower than those in the control rats before 16 weeks. Plasma glucose levels in the metformin-treated rats were lower than those in the control rats, and plasma alanine aminotransferase levels temporarily decreased. The lipid content and some mRNA expression in relation to fibrosis in the liver decreased with pioglitazone treatment, and the mRNA expression of microsomal triglyceride transfer protein increased. Hepatic fibrosis observed in the SDT fatty rats improved with pioglitazone treatment; however, the effect with metformin treatment was partial. These results in both drugs are in line with results in the human study, suggesting that the SDT fatty rat is useful for developing new anti-NASH drugs that show potential to regulate glucose/ lipid metabolism.

KEY WORDS: metformin, NASH, pioglitazone, SDT fatty rat

Nonalcoholic steatohepatitis (NASH) is promoted on the basis of the pathology of nonalcoholic fatty liver disease (NAFLD), and some NASH-patients progress to hepatic cirrhosis and/or carcinoma [3, 21]. NAFLD is associated with metabolic diseases, such as type 2 diabetes, insulin resistance, obesity, and dyslipidemia [2]. The number of patients with metabolic diseases is increasing, and the population of NASH patients is rapidly increasing all over the world. However, the pathophysiology of NASH seems to be poorly defined, and effective pharmacological therapies have not been approved [27].

Experimental animal models play key roles in gaining a better understanding of the pathophysiology and in developing new drugs for NASH. Some animal models have been developed and, in particular, using an animal model based on insulin resistance to investigate NASH is important. The Spontaneously Diabetic Torii (SDT) fatty rat, established by introducing the $f a$ allele of the Zucker fatty rat into the SDT rat genome, is a new model for obese type 2 diabetes [12, 22]. The female SDT fatty rat exhibits obesity, hyperinsulinemia, hyperglycemia and dyslipidemia at approximately 6 weeks of age, and hepatic NASH-like lesions are

*Correspondence to: Ohta, T.: takeshi.ota@jt.com

O2018 The Japanese Society of Veterinary Science

This is an open-access article distributed under the terms of the Creative Commons Attribution Non-Commercial No Derivatives (by-nc-nd) License. (CC-BY-NC-ND 4.0: https://creativecommons.org/licenses/by-nc-nd/4.0/) 
observed at 32-40 weeks of age [8, 9, 23]. Moreover, the NASH-like lesions in female SDT fatty rats are observed at 20 weeks of age with a cholesterol-enriched diet [29]. The female SDT fatty rat is expected to be useful for NASH research. Furthermore, in addition to pathophysiological analyses of NASH in the animal model, investigating the pharmacological effects of anti-diabetic drugs on NASH to elucidate the properties of these animals as a NASH animal model is important. Currently, two anti-diabetic drugs, metformin and pioglitazone are clinically used as NASH therapies; however, both are not officially approved, and this use is off-label. Both drugs reportedly showed the improvement of hepatic lesions in NASH patients; however, there are differences in mechanism and pharmacological potential in both drugs $[6,18]$. Metformin suppresses gluconeogenesis in liver and pioglitazone activates peroxisome proliferator-activated receptor (PPAR)- $\gamma$ in adipose tissues $[14,16]$. In this study, metformin and pioglitazone were repeatedly administered to female SDT fatty rats fed a cholesterol-enriched diet, and the effects of both drugs were investigated.

\section{MATERIALS AND METHODS}

\section{Animals and compounds}

Female SDT fatty rats were purchased from CLEA Japan Inc. (Tokyo, Japan). At four weeks of age, SDT fatty rats were divided into three groups $(\mathrm{n}=6)$; a control group, a metformin treated group, and a pioglitazone treated group. Female SpragueDawley (SD) rats (CLEA Japan Inc.) were used as normal rats $(\mathrm{n}=6)$. Rats were housed in suspended bracket cages and given a cholesterol-enriched powder diet (CRF-1 with $2 \%$ cholesterol added, Oriental Yeast Co., Ltd., Tokyo, Japan) and water ad libitum in a controlled room for temperature $\left(23 \pm 3^{\circ} \mathrm{C}\right)$, humidity $(55 \pm 15 \%)$, and lighting (a 12-hr dark-light cycle). SDT fatty rats were fed a powder diet mixed with pioglitazone $(0.01 \%)$ to achieve a total daily dose of approximately $10 \mathrm{mg} / \mathrm{kg}$ from 4 to $25 \mathrm{weeks}$ of age. Metformin $(0.3 \%)$ was added to a powder diet to achieve a daily dose of approximately $300 \mathrm{mg} / \mathrm{kg}$ from 4 to 25 weeks of age. SDT fatty rats in the control group and SD rats were fed a cholesterol-enriched powder diet from 4 to 25 weeks of age. All animals protocols used in this study were in strict compliance with our own Laboratory Guidelines for Animal Experimentation.

\section{Biological parameters}

Food intake, body weight, and non-fasting serum biochemical parameters, such as plasma glucose, insulin, triglyceride (TG), total cholesterol (TC), alanine aminotransferase (ALT), and aspartate aminotransferase (AST) levels, were evaluated every four weeks, from 4 to 24 weeks of age. Because there were multiple rats in each cage, the food intake was calculated by dividing the total cage food intake by the number of animals per cage. Blood samples were collected from the tail veins of rats at 9-10 a.m. Glucose, TG, TC, ALT and AST levels were measured using commercial kits (Roche Diagnostics, Basel, Switzerland) and an automatic analyzer (Hitachi, Tokyo, Japan). Insulin level was measured using rat-insulin enzyme-linked immunosorbent assay (ELISA) kits (Morinaga Institute of Biological Science, Yokohama, Japan).

\section{Tissue sampling and histopathology}

Necropsies were performed at 25 weeks of age. All animals were sacrificed by exsanguination under isoflurane anesthesia. After measuring liver weights, the livers were sampled for measurement of gene expression, hepatic lipid content, and histopathology. Samples for determination of gene expression and hepatic lipid content were stored at $-80^{\circ} \mathrm{C}$ until analysis. For histopathology, the livers were immediately fixed in $10 \%$ neural-buffered formalin. After resection, the tissues were paraffin-embedded using standard techniques and thin-sectioned ( 3 to $5 \mu \mathrm{m}$ ). The sections were stained with hematoxylin and eosin (HE) and Sirius Red.

\section{Hepatic lipid contents}

An approximately $100 \mathrm{mg}$ portion of the liver, $0.5 \mathrm{ml}$ of methanol, and zirconia beads were added to tubes. The liver portion was homogenized using a mixer mill (MM300 Retch) $(25 \mathrm{~Hz}, 10 \mathrm{~min})$. To the homogenized solution, $1 \mathrm{~m} l$ of chloroform was added and mixed thoroughly. The mixture was then centrifuged $\left(10,000 \mathrm{~g}, 5 \mathrm{~min}, 4^{\circ} \mathrm{C}\right)$ and the resulting supernatant collected. Solvents contained in $0.5 \mathrm{~m} l$ of the supernatant were dried under a stream of nitrogen gas. To the residue, $0.5 \mathrm{~m} l$ of 2 -propanol was added, and the residue was then dissolved again. TG, TC and free fatty acid (FFA) concentrations of the 2-propanol solution were determined using a biochemistry automatic analyzer (Hitachi 7170S; Hitachi, Tokyo, Japan). TG, TC and FFA levels were measured using commercial kits (Roche Diagnostics, Basel, Switzerland).

\section{mRNA quantification with real-time quantitative PCR}

Total RNA was extracted from the liver at 25 weeks of age using the miRNeasy Mini Kit (Qiagen, Hilden, Germany) according to the manufacturer's protocols. Complementary DNA (cDNA) was synthesized from $1 \mu \mathrm{g}$ of total RNA using a High-Capacity cDNA Reverse Transcription Kit with an RNase Inhibitor (Applied Biosystems, Foster City, CA, U.S.A.). The reaction mixture was incubated for $10 \mathrm{~min}$ at $25^{\circ} \mathrm{C}, 2 \mathrm{hr}$ at $37^{\circ} \mathrm{C}$, and $5 \mathrm{~min}$ at $85^{\circ} \mathrm{C}$. Real-time PCR quantification was performed in a $20 \mu l$ reaction mixture on a QuantStudio 3 Real-Time PCR system (Applied Biosystems). The reaction mixture contained 1× TaqMan Universal PCR Master Mix II (Applied Biosystems), $50 \mathrm{ng}$ of synthesized cDNA, and $0.9 \mu \mathrm{M}$ primers $/ 0.25 \mu \mathrm{M}$ probes or TaqMan primers/probe mix (TaqMan Gene Expression Assays, Applied Biosystems). Cycle parameters were 10 min at $95^{\circ} \mathrm{C}$, followed by 40 cycles of $15 \mathrm{sec}$ at $95^{\circ} \mathrm{C}$ and $1 \mathrm{~min}$ at $60^{\circ} \mathrm{C}$. The following primers and FAM-conjugated probe were designed using Primer Express software (Applied Biosystems): fatty acid synthase (FAS) (forward, ACTGAACGGCATTACTCGGTCC; reverse, GTGTCCCATGTTGGATTTGGTG; probe, TTCCGCCAGAGCCCTTTGTTAATTGG), acetyl-CoA carboxylase 
(A)

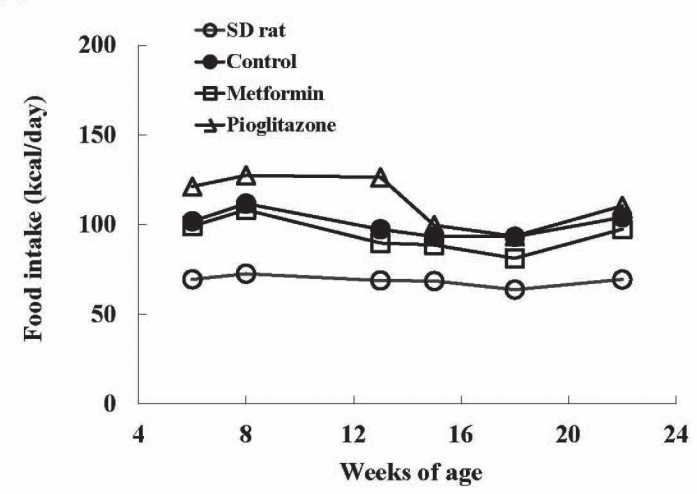

(B)

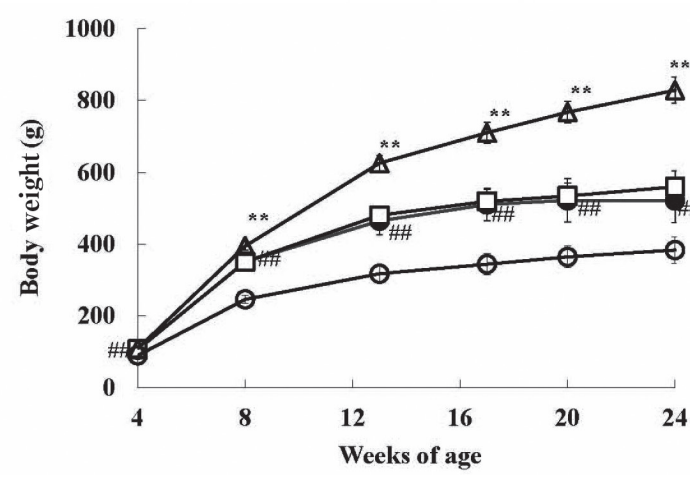

Fig. 1. Effects of metformin and pioglitazone on food intake (A) and body weight (B) in female SDT fatty rats fed a cholesterol-enriched diet. (A) Data represent mean values ( $\mathrm{n}=6$ ). (B) Data represent means \pm standard deviation $(\mathrm{n}=6)$. ${ }^{* *} P<0.01$; significantly different from the control group. $\# \# P<0.01$; significantly different from the $\mathrm{SD}$ rat group.

(ACC) (forward, GCAGCTATGTTCAGAGAGTTCACC; reverse, CCACCTCACAGTTGACTTGTTTTC; probe, CGGCGACTTACGTTCCTAGTTGCACAAAA). Expression of the following genes was confirmed using TaqMan Gene Expression Assays: $\beta$-actin (Rn00667869_m1), tumor necrosis factor (TNF)- $\alpha$ (Rn99999017_m1), monocyte chemoattractant protein (MCP)-1 (Rn00580555_m1), transforming growth factor (TGF)- $\beta$ (Rn99999016_m1), collagen type I $\alpha-1$ (COLIA1) (Rn01463848_m1), $\alpha$-smooth muscle actin (SMA) (Rn01759928_g1), and microsomal triglyceride transfer protein (MTP) (Rn01522970_m1).

\section{Statistical analysis}

The results of biological parameters except for food intake are expressed as means \pm standard deviation. Food intake is expressed in mean values. Statistical analyses of differences between mean values were performed using a Tukey Kramer test. Differences were considered significant at $P<0.05$.

\section{RESULTS}

Changes in food intake and body weight are shown in Fig. 1. Food intake in the control group was higher than that in the SD rat group, and food intake in the pioglitazone group was higher than in the control group (Fig. 1A). Control female SDT fatty rats showed overt obesity, and body weight in the pioglitazone group was significantly higher than that in the control group; however, body weight in the metformin group was comparable to that in the control group (Fig. 1B).

Changes in biochemical parameters are shown in Fig. 2. Control female SDT fatty rats showed hyperglycemia, hyperinsulinemia, and hyperlipidemia, and the plasma ALT and AST levels also increased compared with the SD rat group during the experimental period. In the pioglitazone group, increases in plasma glucose, TG, TC, ALT and AST levels were significantly suppressed during the experimental period, the glucose and TG levels were reduced to similar levels observed in the SD rat group. Moreover, plasma insulin level in the pioglitazone group was significantly lower than that in the control group at 12 weeks of age (Fig. 2B). In the metformin group, the increased plasma glucose levels significantly decreased compared with the control group (Fig. 2A), and the increasing in plasma ALT levels temporarily decreased (Fig. 2C and 2E). The metformin treatment did not affect plasma insulin, TC and AST levels.

Liver weight in the control group showed a significant increase compared with that in the SD rat group, and liver weight in the pioglitazone group decreased compared with that in the control group; however, the weight in the metformin group was comparable to that in the control group (Relative liver weights at 25 weeks of age: control group; $82.6 \pm 13.7 \mathrm{mg} / \mathrm{g}$, metformin group; $78.9 \pm$ $21.5 \mathrm{mg} / \mathrm{g}$, pioglitazone group; $23.6 \pm 2.2 \mathrm{mg} / \mathrm{g}$, SD rat group; $28.2 \pm 2.4 \mathrm{mg} / \mathrm{g}$ ). Hepatic lipid content in the control group showed a remarkable increase compared with that in the SD rat group, and hepatic lipid content in the pioglitazone group significantly decreased compared with that in the control group; however, the content in the metformin group was comparable to that in the control group (Fig. 3).

The liver histopathology was examined by HE staining and Sirius Red staining to evaluate fibrosis (Table 1). Representative histological photographs in each group are shown in Fig. 4. In the control group, moderate changes in hepatosteatosis, moderate or severe changes in hypertrophic hepatocytes, and slight or moderate changes in inflammation and fibrosis were observed (Fig. 4B and 4F). In the pioglitazone group, the hepatosteatosis and slightly or very slightly hypertrophic hepatocytes were observed, although some rats did not show histological changes including inflammation and fibrosis (Fig. 4D and 4H). In the metformin group, only one rat (No. 13) showed improvements in hepatic abnormalities.

Changes in mRNA expression related to inflammation, fibrosis, and lipid synthesis were determined in each group (Fig. 5). In the control group, the mRNA expression of COLIA1, a fibrosis-related factor, and ACC/FAS, lipid synthesis-related factors, 
(A)

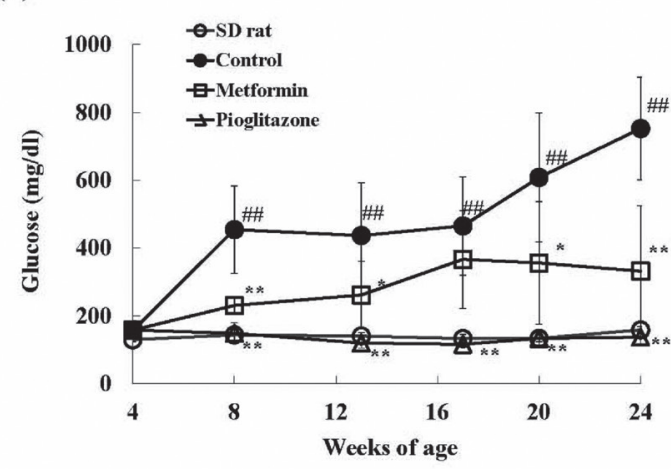

(B)

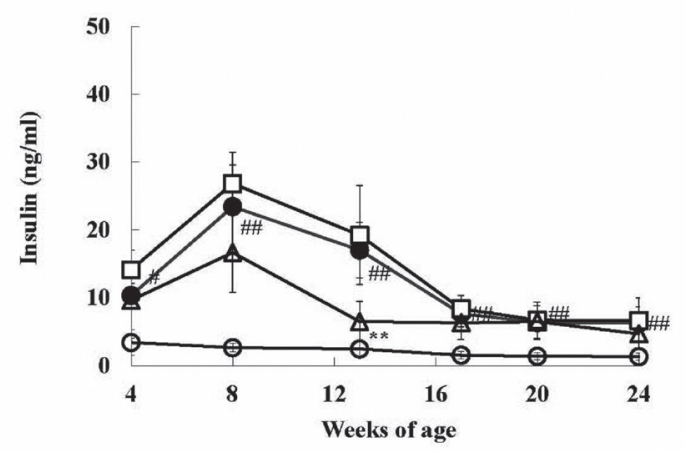

(C)

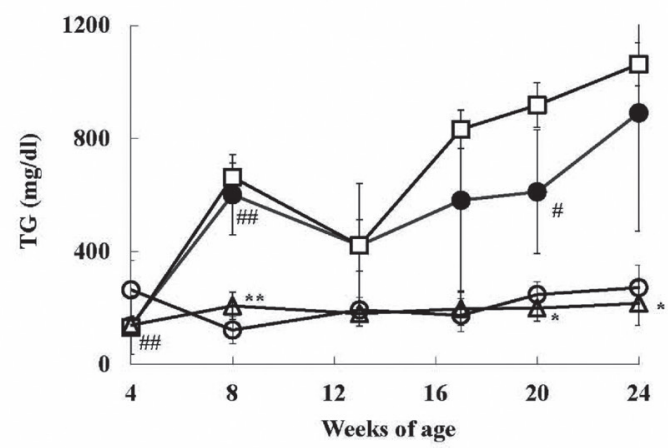

(D)

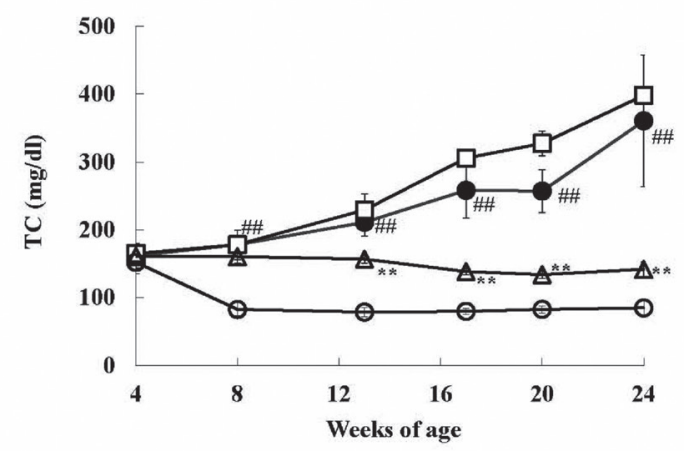

(E)

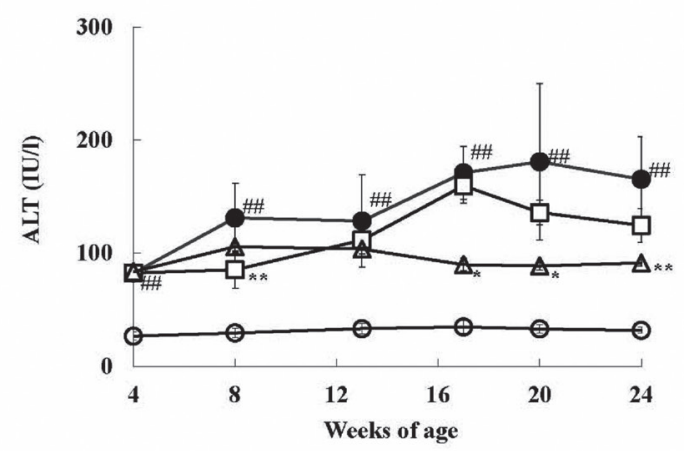

(F)

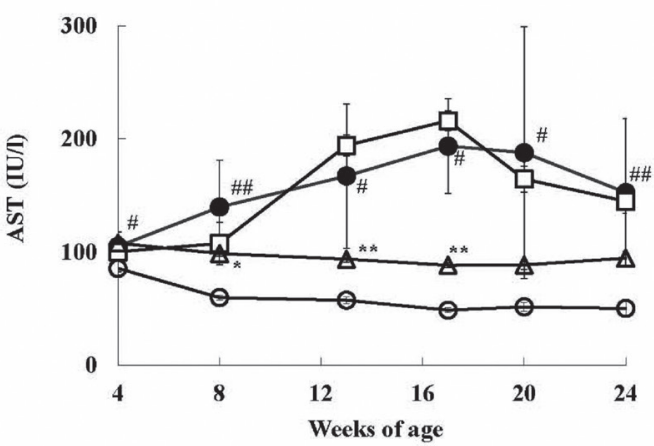

Fig. 2. Effects of metformin and pioglitazone on plasma glucose (A), insulin (B), triglyceride (TG) (C), total cholesterol (TC) (D), alanine aminotransferase (ALT) (E), and aspartate aminotransferase (AST) (F) levels in female SDT fatty rats fed a cholesterol-enriched diet. Data represent means \pm standard deviation $(\mathrm{n}=6)$. $* P<0.05$, $* * P<0.01$; significantly different from the control group. $\# P<0.05$, \#\#P<0.01; significantly different from the SD rat group.

(A)

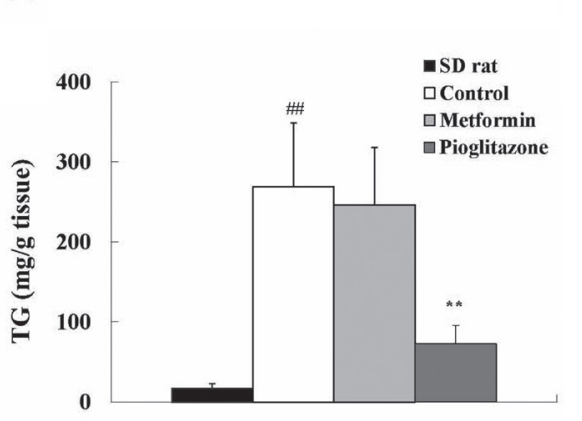

(B)

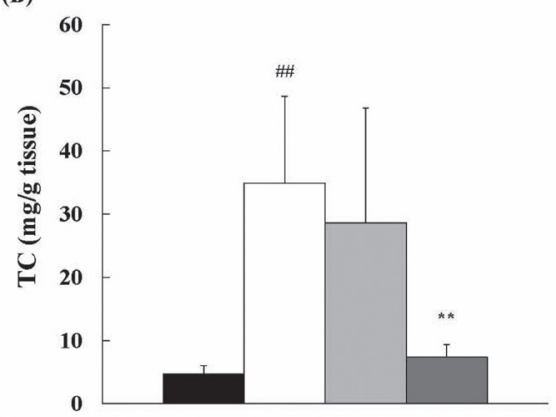

(C)

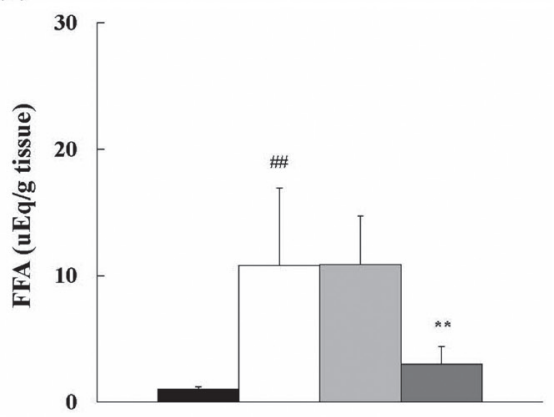

Fig. 3. Changes in hepatic triglyceride (TG) (A), total cholesterol (TC) (B), and free fatty acid (FFA) (C) contents in female SDT fatty rats fed a cholesterol-enriched diet. Data represent means \pm standard deviation $(\mathrm{n}=6) .{ }^{*} P<0.01$; significantly different from the control group. \#\#P<0.01; significantly different from the SD rat group. 
Table 1. Histological findings of livers in cholesterol-loaded SDT fatty rats that received chronic treatment with anti-diabetic drugs

\begin{tabular}{|c|c|c|c|c|c|c|c|c|c|c|c|c|c|c|c|c|c|c|c|c|c|c|c|c|}
\hline \multirow{3}{*}{ Findings } & \multirow{3}{*}{$\begin{array}{c}\text { Animal } \\
\text { No. }\end{array}$} & \multicolumn{23}{|c|}{25 weeks of age } \\
\hline & & \multicolumn{6}{|c|}{ SD rat } & \multicolumn{6}{|c|}{ Control } & \multicolumn{5}{|c|}{ Metformin } & \multicolumn{6}{|c|}{ Pioglitazone } \\
\hline & & 1 & 2 & 3 & 4 & 5 & 6 & 7 & 8 & 9 & 10 & 11 & 12 & 13 & 14 & 1516 & 17 & 18 & 19 & 20 & 21 & 22 & 23 & 24 \\
\hline Fatty change & & - & - & - & - & - & - & $3+$ & $3+$ & $3+$ & $3+$ & $3+$ & $3+$ & $2+$ & $3+$ & $3+3+$ & $3+$ & $3+$ & + & + & + & + & \pm & + \\
\hline Hypertrophy, hepatocyte & & - & - & - & - & - & - & $2+$ & $2+$ & $2+$ & $2+$ & $2+$ & $3+$ & + & $2+$ & $3+3+$ & $2+$ & $2+$ & \pm & \pm & \pm & \pm & \pm & \pm \\
\hline Infiltration, inflammatory cell, periportal & & - & - & - & - & - & \pm & + & $2+$ & + & + & \pm & $2+$ & \pm & + & $2++$ & + & \pm & - & + & \pm & \pm & - & - \\
\hline Fibrosis, focal & & - & - & - & - & - & - & + & + & $2+$ & $2+$ & - & + & \pm & $2+$ & $+\quad+$ & + & \pm & \pm & \pm & - & - & - & \pm \\
\hline
\end{tabular}

-: Negative, \pm : Very slight, +: Slight, 2+: Moderate, 3+: Severe.
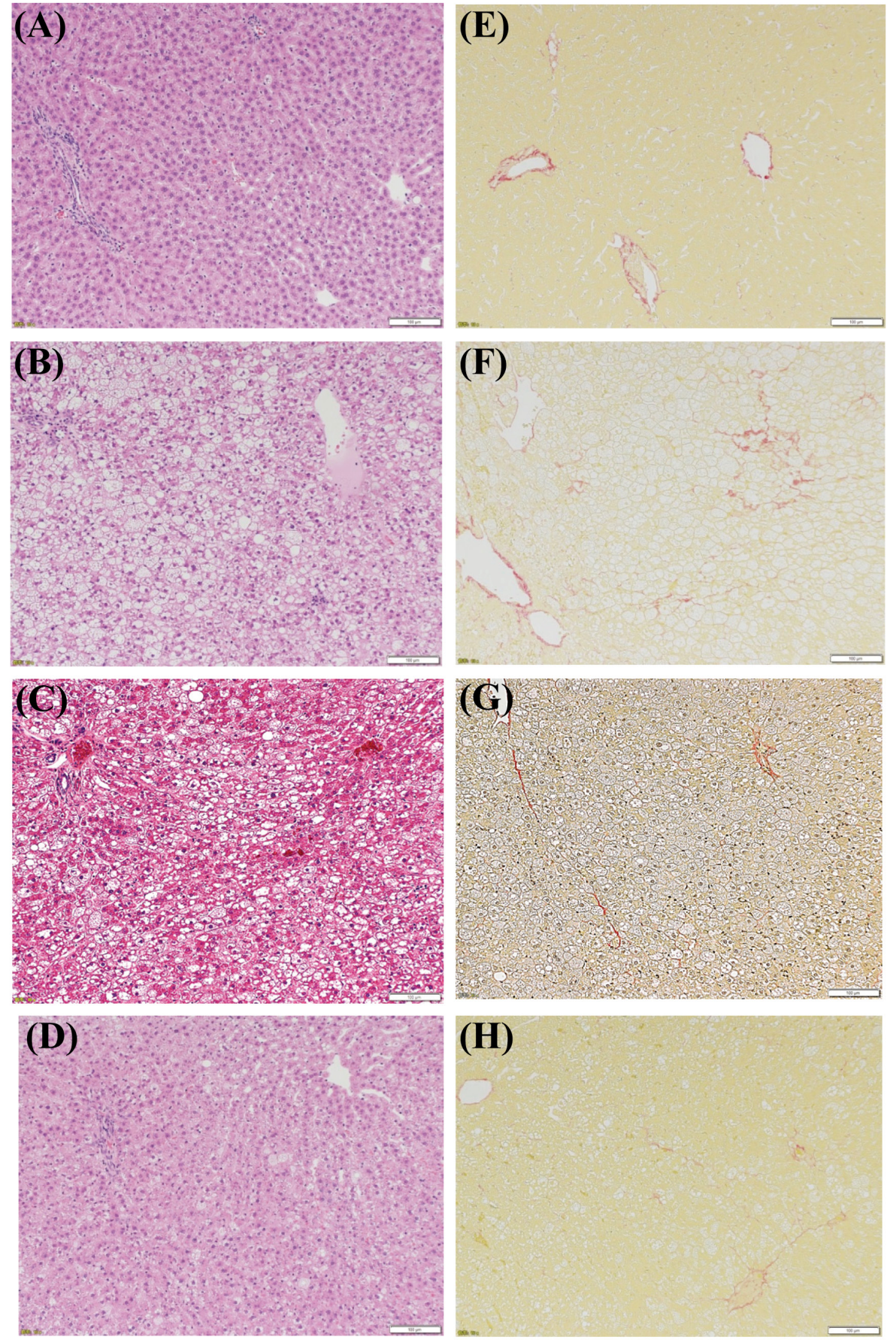

Fig. 4. Liver histopathology at 25 weeks of age. (A, E): the SD group (animal No. 6); (B, F): the control group (animal No. 7); (C, G): the metformin group (animal No. 18); (D, H): the pioglitazone group (animal No. 19). (A-D): Hematoxylin and eosin (HE) staining. (E-H): Sirius red staining. Bar $=100 \mu \mathrm{m}$. 
(A)

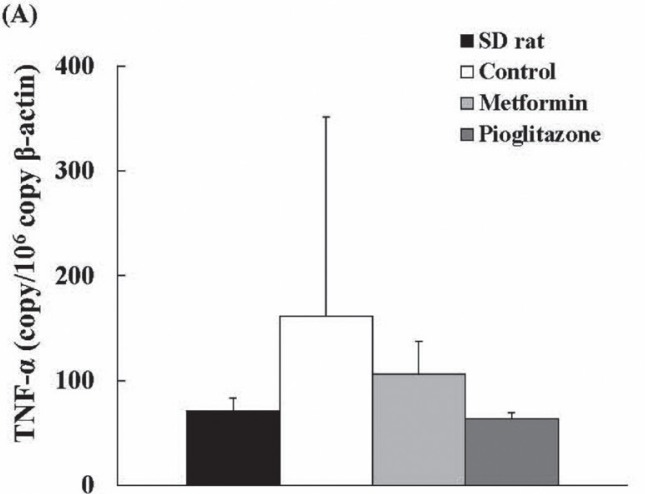

(B)

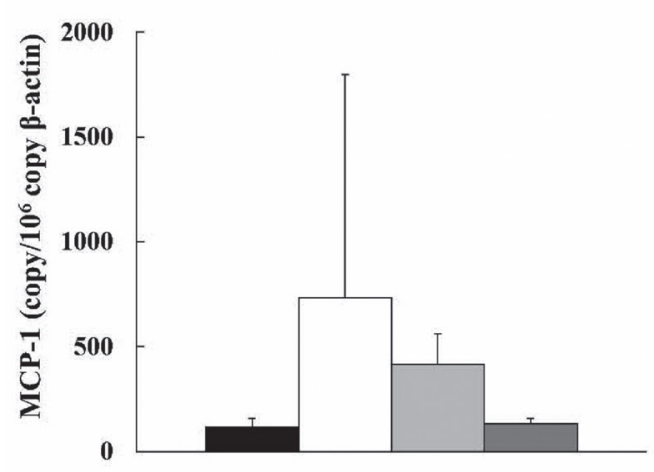

(C)

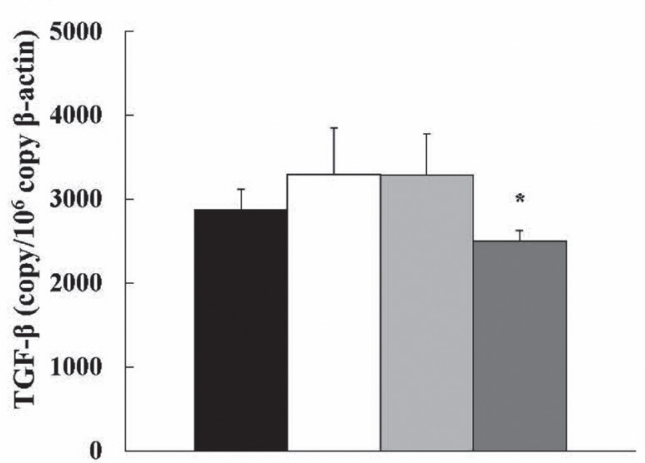

(D)

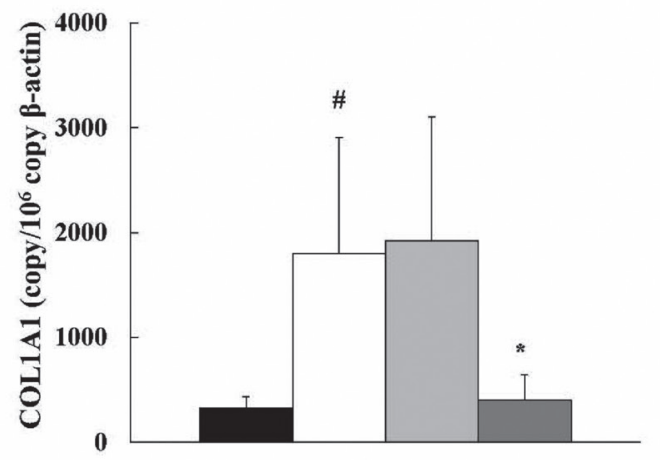

(E)

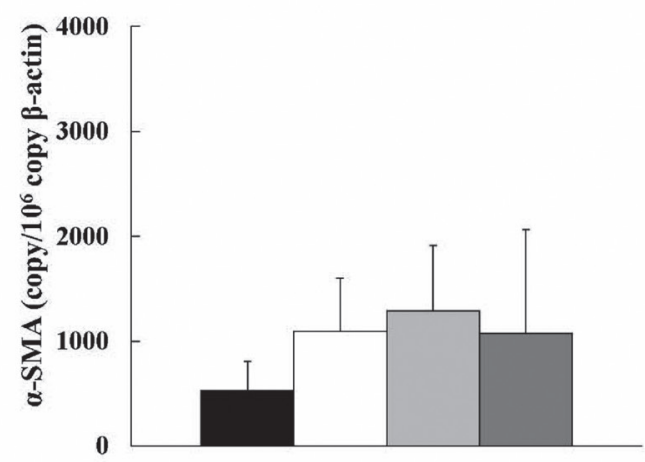

(F)

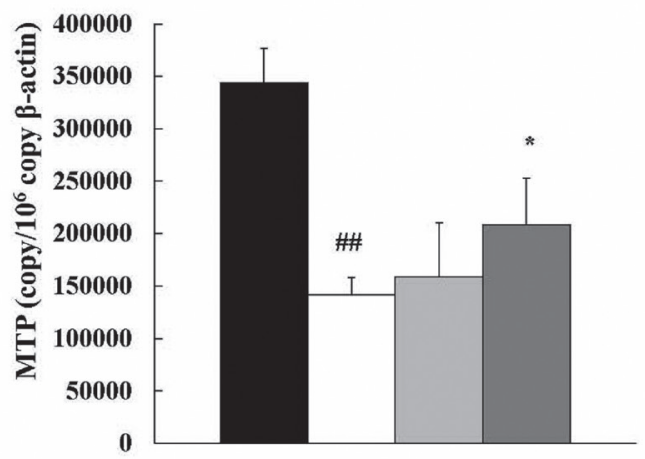

(G)

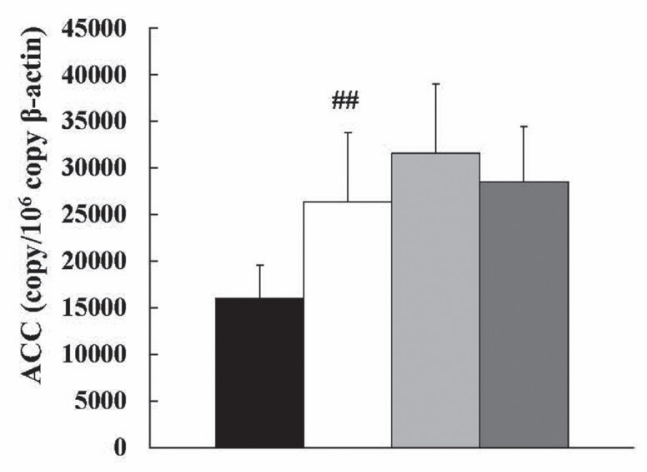

(H)

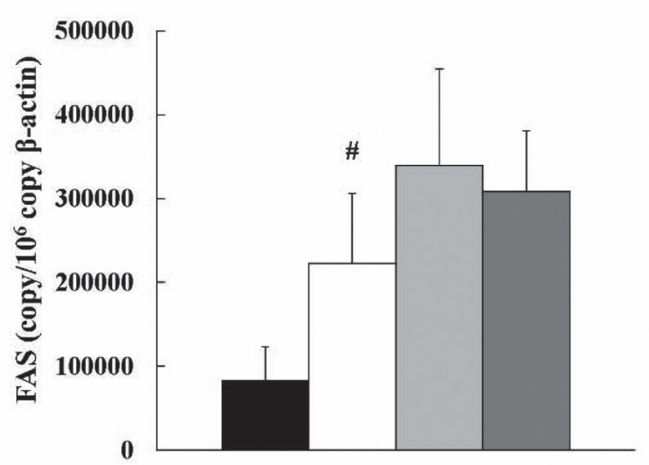

Fig. 5. Changes in hepatic tumor necrosis factor (TNF)- $\alpha(\mathrm{A})$, monocyte chemotactic protein (MCP)-1 (B), transforming growth factor (TGF)- $\beta$ (C), collagen type I $\alpha 1$ (COLIA1) (D), $\alpha$-smooth muscle actin (SMA) (E), microsomal triglyceride transfer protein (MTP) (F), acetyl-CoA carboxylase (ACC) (G), and fatty acid synthase (FAS) (H) mRNA expression in female SDT fatty rats fed a cholesterol-enriched diet. Data represent means \pm standard deviation $(\mathrm{n}=6) . * P<0.05$; significantly different from the control group. $\# P<0.05, \# \# P<0.01$; significantly different from the SD rat group. 
increased compared with those in the SD rat group, and the mRNA expression of MTP decreased. In the pioglitazone group, the mRNA expression of TGF- $\beta$ and COLIA1 significantly decreased, and the mRNA expression of MTP increased. In the metformin group, no significant changes were detected in the mRNA expression.

\section{DISCUSSION}

NASH is a concerning hepatic disease that occasionally leads to cirrhosis and/or carcinoma [3, 21]. Some theories, such as the "two-hit theory" and "multiple parallel-like theory", have advanced as proposed methods of development of NASH [4, 10, 28]. In either of these theories, lipid synthesis/accumulation and inflammation are important for the development of NAFLD/NASH, and insulin resistance is a basic factor [2]. The female SDT fatty rat used in this study showed NASH-like lesions with insulin resistance, and the rat is expected to be useful as a NASH model $[8,29]$.

In the preliminary study using female SDT fatty rats fed a standard diet, pioglitazone at $3 \mathrm{mg} / \mathrm{kg}$ tended to improve the hyperglycemia, but this did not reach significance (unpublished data), and the dosage of $10 \mathrm{mg} / \mathrm{kg}$ of pioglitazone was used in this study. Pioglitazone treatment prominently suppressed the hyperglycemia and the hyperlipidemia in female SDT fatty rats fed a cholesterol-enriched diet and, furthermore, the plasma insulin levels in pioglitazone treatment decreased, suggesting improvements in insulin sensitivity in the rats. The reduction of hepatic lipid accumulation in the pioglitazone group is considered to be induced by the suppression of hyperglycemia and hyperlipidemia $[1,11]$. Moreover, the increase in hepatic expression level of MTP leads to the reduction of hepatic lipid accumulation. The suppression of MTP expression reportedly induces hepatic fat accumulation [17]. The decreases in hepatic lipid levels are considered as suppressing the inflammation and/or fibrosis in the liver with pioglitazone treatment. Increases in hepatic lipid levels, such as FFA and cholesterol, reportedly result in the development of inflammation and/or fibrosis in the liver $[7,24,26]$. The decreases in plasma ALT and AST levels are also considered to be related to improvements in hepatic lesions. In pioglitazone treatment, however, body weight gain was observed along with improvements in hepatic lesions. In clinical studies, pioglitazone treatment also induced body weight gain along with therapeutic effects on NASH $[6,19]$. Pioglitazone treatment induces a lipid accumulation in adipose tissues via a regulation of PPAR- $\gamma$ activity. Diet control is reportedly important with therapy using pioglitazone. The therapeutic effects with pioglitazone treatment were reportedly correlated with an improvement of insulin sensitivity [5]. Actually, in this study, pioglitazone treatment suppressed the hyperinsulinemia in SDT fatty rats.

Since the plasma ALT and AST levels increased at $300 \mathrm{mg} / \mathrm{kg}$ or higher in the preliminary study (unpublished data), the dosage of $300 \mathrm{mg} / \mathrm{kg}$ of metformin was used in this study. Metformin treatment significantly inhibited the hyperglycemia in SDT fatty rats fed a cholesterol-enriched diet; however, the pharmacological potential to improve NASH-like lesions is mild when compared with pioglitazone treatment. Moreover, metformin treatment did not result in an improvement in insulin sensitivity. The pharmacological effects of metformin on NASH were considered to be partial because of the insufficient effects on blood glucose/lipid control and insulin resistance. On the other hand, it is reported that metformin improves hepatic insulin signaling and histological damages of liver in insulin-resistant rats of NASH and cirrhosis [30]. In clinical studies, the pharmacological potential to improve NASH with metformin treatment is weak when compared with pioglitazone treatment, and the drug leads to improvement in hepatic histology and ALT levels in only $30 \%$ of patients with NASH. Moreover, the histological improvement did not correlate with the degree of changes in insulin sensitivity $[18,20]$. The pharmacological effects of both drugs on SDT fatty rats are considered to be reflective of the results in clinical studies. On the other hand, currently, numerous candidate compounds, such as farnesoid X receptor (FXR) agonists, PPAR $\alpha / \delta$ agonists, and agents targeting inflammation, are developing for NASH therapy [25]. In future, it is necessary to evaluate the pharmacological effects of those compounds on SDT fatty rats.

The pharmacological effects of pioglitazone on NASH treatment have been reported in some animal models, such as the methionine-choline deficient (MCD) diet-induced model and STAM mice. In those models, pioglitazone treatment improved hepatic NASH-like lesions, but did not result in changes in body weight $[13,15]$. Animal models demonstrating obesity and insulin resistance are essential for the pharmacological assessment of anti-NASH drugs.

In female SDT fatty rats fed a cholesterol-enriched diet, pioglitazone improved hepatic lesions; however, the body weight increased. The effects of metformin on hepatic lesions were partial. The SDT fatty rats showed different pharmacological responses to two kinds of anti-diabetic drugs, metformin and pioglitazone. These results in both drugs are similar to what is observed in human studies, suggesting that the SDT fatty rat is useful for the development of new anti-NASH drugs that show potential to regulate glucose/lipid metabolism.

CONFLICT OF INTEREST. Toriniwa, Saito, Miyajima, Ishii, Maekawa and Ohta are employees of Japan Tobacco Inc.

\section{REFERENCES}

1. Day, C. P. and James, O. F. 1998. Steatohepatitis: a tale of two "hits"? Gastroenterology 114: 842-845. [Medline] [CrossRef]

2. de Alwis, N. M. and Day, C. P. 2008. Non-alcoholic fatty liver disease: the mist gradually clears. J. Hepatol. 48 Suppl 1: S104-S112. [Medline] [CrossRef]

3. Ertle, J., Dechêne, A., Sowa, J. P., Penndorf, V., Herzer, K., Kaiser, G., Schlaak, J. F., Gerken, G., Syn, W. K. and Canbay, A. 2011. Non-alcoholic fatty liver disease progresses to hepatocellular carcinoma in the absence of apparent cirrhosis. Int. J. Cancer 128: 2436-2443. [Medline] [CrossRef]

4. Fukunishi, S., Nishio, H., Fukuda, A., Takeshita, A., Hanafusa, T., Higuchi, K. and Suzuki, K. 2009. Development of Fibrosis in Nonalcoholic 
Steatosis through Combination of a Synthetic Diet Rich in Disaccharide and Low-Dose Lipopolysaccharides in the Livers of Zucker (fa/fa) Rats. $J$. Clin. Biochem. Nutr. 45: 322-328. [Medline] [CrossRef]

5. Gastaldelli, A., Harrison, S. A., Belfort-Aguilar, R., Hardies, L. J., Balas, B., Schenker, S. and Cusi, K. 2009. Importance of changes in adipose tissue insulin resistance to histological response during thiazolidinedione treatment of patients with nonalcoholic steatohepatitis. Hepatology $\mathbf{5 0}$ 1087-1093. [Medline] [CrossRef]

6. He, L., Liu, X., Wang, L. and Yang, Z. 2016. Thiazolidinediones for nonalcoholic steatohepatitis: A meta-analysis of randomized clinical trials. Medicine (Baltimore) 95: e4947. [Medline] [CrossRef]

7. Ichimura, M., Kawase, M., Masuzumi, M., Sakaki, M., Nagata, Y., Tanaka, K., Suruga, K., Tamaru, S., Kato, S., Tsuneyama, K. and Omagari, K. 2015. High-fat and high-cholesterol diet rapidly induces non-alcoholic steatohepatitis with advanced fibrosis in Sprague-Dawley rats. Hepatol. Res. 45: 458-469. [Medline] [CrossRef]

8. Ishii, Y., Motohashi, Y., Muramatsu, M., Katsuda, Y., Miyajima, K., Sasase, T., Yamada, T., Matsui, T., Kume, S. and Ohta, T. 2015. Female spontaneously diabetic Torii fatty rats develop nonalcoholic steatohepatitis-like hepatic lesions. World J. Gastroenterol. 21: 9067-9078. [Medline] [CrossRef]

9. Ishii, Y., Ohta, T., Sasase, T., Morinaga, H., Ueda, N., Hata, T., Kakutani, M., Miyajima, K., Katsuda, Y., Masuyama, T., Shinohara, M. and Matsushita, M. 2010. Pathophysiological analysis of female Spontaneously Diabetic Torii fatty rats. Exp. Anim. 59: 73-84. [Medline] [CrossRef]

10. Ito, M., Suzuki, J., Sasaki, M., Watanabe, K., Tsujioka, S., Takahashi, Y., Gomori, A., Hirose, H., Ishihara, A., Iwaasa, H. and Kanatani, A. 2006. Development of nonalcoholic steatohepatitis model through combination of high-fat diet and tetracycline with morbid obesity in mice. Hepatol. Res. 34: 92-98. [Medline] [CrossRef]

11. Karim, M. F., Al-Mahtab, M., Rahman, S. and Debnath, C. R. 2015. Non-alcoholic Fatty Liver Disease (NAFLD)--A Review. Mymensingh Med. J. 24: 873-880. [Medline]

12. Katsuda, Y., Ohta, T., Miyajima, K., Kemmochi, Y., Sasase, T., Tong, B., Shinohara, M. and Yamada, T. 2014. Diabetic complications in obese type 2 diabetic rat models. Exp. Anim. 63: 121-132. [Medline] [CrossRef]

13. Kawai, D., Takaki, A., Nakatsuka, A., Wada, J., Tamaki, N., Yasunaka, T., Koike, K., Tsuzaki, R., Matsumoto, K., Miyake, Y., Shiraha, H., Morita, M., Makino, H. and Yamamoto, K. 2012. Hydrogen-rich water prevents progression of nonalcoholic steatohepatitis and accompanying hepatocarcinogenesis in mice. Hepatology 56: 912-921. [Medline] [CrossRef]

14. Lebovitz, H. E. and Banerji, M. A. 2001. Insulin resistance and its treatment by thiazolidinediones. Recent Prog. Horm. Res. 56: $265-294$. [Medline] [CrossRef]

15. Leclercq, I. A., Lebrun, V. A., Stärkel, P. and Horsmans, Y. J. 2007. Intrahepatic insulin resistance in a murine model of steatohepatitis: effect of PPARgamma agonist pioglitazone. Lab. Invest. 87: 56-65. [Medline] [CrossRef]

16. Link, J. T. 2003. Pharmacological regulation of hepatic glucose production. Curr. Opin. Investig. Drugs 4: 421-429. [Medline]

17. Lin, M., Zhao, S., Shen, L. and Xu, D. 2014. Potential approaches to ameliorate hepatic fat accumulation seen with MTP inhibition. Drug Saf. 37: 213-224. [Medline] [CrossRef]

18. Li, Y., Liu, L., Wang, B., Wang, J. and Chen, D. 2013. Metformin in non-alcoholic fatty liver disease: A systematic review and meta-analysis. Biomed. Rep. 1: 57-64. [Medline] [CrossRef]

19. Lomonaco, R., Sunny, N. E., Bril, F. and Cusi, K. 2013. Nonalcoholic fatty liver disease: current issues and novel treatment approaches. Drugs $\mathbf{7 3}$ 1-14. [Medline] [CrossRef]

20. Loomba, R., Lutchman, G., Kleiner, D. E., Ricks, M., Feld, J. J., Borg, B. B., Modi, A., Nagabhyru, P., Sumner, A. E., Liang, T. J. and Hoofnagle, J. H. 2009. Clinical trial: pilot study of metformin for the treatment of non-alcoholic steatohepatitis. Aliment. Pharmacol. Ther. 29: $172-182$. [Medline] [CrossRef]

21. Malik, S. M., Gupte, P. A., de Vera, M. E. and Ahmad, J. 2009. Liver transplantation in patients with nonalcoholic steatohepatitis-related hepatocellular carcinoma. Clin. Gastroenterol. Hepatol. 7: 800-806. [Medline] [CrossRef]

22. Masuyama, T., Katsuda, Y. and Shinohara, M. 2005. A novel model of obesity-related diabetes: introgression of the Lepr(fa) allele of the Zucker fatty rat into nonobese Spontaneously Diabetic Torii (SDT) rats. Exp. Anim. 54: 13-20. [Medline] [CrossRef]

23. Matsui, K., Ohta, T., Oda, T., Sasase, T., Ueda, N., Miyajima, K., Masuyama, T., Shinohara, M. and Matsushita, M. 2008. Diabetes-associated complications in Spontaneously Diabetic Torii fatty rats. Exp. Anim. 57: 111-121. [Medline] [CrossRef]

24. Neuschwander-Tetri, B. A. 2010. Hepatic lipotoxicity and the pathogenesis of nonalcoholic steatohepatitis: the central role of nontriglyceride fatty acid metabolites. Hepatology 52: 774-788. [Medline] [CrossRef]

25. Oseini, A. M. and Sanyal, A. J. 2017. Therapies in non-alcoholic steatohepatitis (NASH). Liver Int. 37 Suppl 1: 97-103. [Medline] [CrossRef]

26. Pettinelli, P., Obregón, A. M. and Videla, L. A. 2011. Molecular mechanisms of steatosis in nonalcoholic fatty liver disease. Nutr. Hosp. 26: 441-450. [Medline]

27. Sanyal, A. J., Friedman, S. L., McCullough, A. J., Dimick-Santos L. and American Association for the Study of Liver Diseases United States Food and Drug Administration. 2015. Challenges and opportunities in drug and biomarker development for nonalcoholic steatohepatitis: findings and recommendations from an American Association for the Study of Liver Diseases-U.S. Food and Drug Administration Joint Workshop. Hepatology 61: $1392-405$.

28. Takaki, A., Kawai, D. and Yamamoto, K. 2013. Multiple hits, including oxidative stress, as pathogenesis and treatment target in non-alcoholic steatohepatitis (NASH). Int. J. Mol. Sci. 14: 20704-20728. [Medline] [CrossRef]

29. Toriniwa, Y., Muramatsu, M., Ishii, Y., Riya, E., Miyajima, K., Ohshida, S., Kitatani, K., Takekoshi, S., Matsui, T., Kume, S., Yamada, T. and Ohta, T. 2018. Pathophysiological Characteristics of Non-Alcoholic Steatohepatitis-like Changes in Cholesterol-Loaded Type 2 Diabetic Rats. Physiol. Res. (in press).

30. Xu, H., Zhou, Y., Liu, Y., Ping, J., Shou, Q., Chen, F. and Ruo, R. 2016. Metformin improves hepatic IRS2/PI3K/Akt signaling in insulin-resistant rats of NASH and cirrhosis. J. Endocrinol. 229: 133-144. [Medline] [CrossRef] 